\title{
Low-grade serous carcinoma of the ovary: A case series and literature review
}

\author{
Joel Alcid, Mark S. Shahin
}

\begin{abstract}
Introduction: Low-grade serous ovarian cancer is a rare subtype of epithelial ovarian cancer that is generally resistant to cytotoxic chemotherapy. We present a case series of patients diagnosed with low-grade serous ovarian carcinoma and review of the literature. Serous carcinoma represents the most common histologic subtype of epithelial ovarian cancer. Low-grade serous carcinoma of the ovary may occur de novo or following a diagnosis of serous tumor of low malignant potential. The primary modality of treatment for these patients is surgery. For patients with stage IIIV low-grade serous carcinoma, the standard is maximum cytoreductive surgery, with the goal of achieving minimal residual disease, followed by chemotherapy. Although lowgrade serous carcinoma of the ovary carries a relatively good prognosis, over $80 \%$ of patients will experience disease recurrence. Case Series: We report four cases of patients ages 31-58, with a diagnosis of low-grade serous ovarian cancer. Our patients were treated with cytoreductive surgery, chemotherapy and hormonal therapy. Two of our patients were also enrolled in a MEK inhibitor clinical trial. We describe in detail the clinical course of each patient and provide
\end{abstract}

Joel Alcid ${ }^{1}$, Mark S. Shahin ${ }^{2}$

Affiliations: ${ }^{1}$ Resident, Internal Medicine, Drexel, Philadelphia, PA, USA; ${ }^{2}$ Attending, Gynecologic Oncology, AbingtonJefferson Health Hanjani Institute for Gynecologic Oncology, Abington, PA, USA.

Corresponding Author: Joel Alcid, Hahnemann University Hospital / Drexel Medicine, 239 N Broad St, Philadelphia PA, USA, 19102; Email: joelalcidmd@gmail.com

Received: 27 March 2018

Accepted: 18 May 2018

Published: 09 June 2018 a review of low-grade serous ovarian cancer. Conclusion: Low-grade serous ovarian cancer is a rare subtype of epithelial ovarian cancer with a relatively good prognosis and high rate of disease recurrence. Due to the expression of estrogen receptors on these tumors, hormonal treatment has been shown to decrease the risk of recurrence compared with surveillance. Targeted therapy for recurrent disease has also been shown to have promising results.

Keywords: Chemotherapy, Hormonal therapy, Low-grade serous carcinoma, Ovarian cancer

\section{How to cite this article}

Alcid J, Shahin MS. Low-grade serous carcinoma of the ovary: A case series and literature review. J Case Rep Images Oncology 2018;4:100052Z10JA2018.

Article ID: 100052Z10JA2018

$$
* * * * * * * * *
$$

doi: 10.5348/100052Z10JA2018CS

\section{INTRODUCTION}

Low-grade serous carcinoma of the ovary, now a distinctly recognized sub-category of epithelial ovarian cancer, represents $10 \%$ of all serous epithelial ovarian cancer. Low-grade serous carcinoma of the ovary may occur de novo or following a diagnosis of serous tumor of low malignant potential. The median age at diagnosis is 43 to 46.5 years of age $[1,3]$. Low-grade serous carcinoma has been known to have relative chemoresistance compared to high-grade serous carcinoma. The primary treatment is cytoreductive surgery, followed by platinum/taxane chemotherapy. Some of these tumors express estrogen and progesterone receptors, making hormonal treatment for maintenance therapy an effective choice, and has 


\section{EDORiUM Journals}

J Case Rep Images Oncology 2018;4:100052Z10JA2018.

www.edoriumjournals.com/case-reports/jcro

Alcid et al. 2

shown to decrease the risk of recurrence compared with surveillance. Targeted therapy against those tumors with a mutation in the MAP kinase pathway for recurrent disease has also been shown to have promising results [46]. We present a case series on two patients diagnosed with low-grade serous carcinoma of the ovary.

\section{CASE SERIES}

\section{Case 1}

A 55-year-old female with no significant $\mathrm{PMH}$ and who had been postmenopausal for five years, presented with the complaint of abdominal pain, bloating, nausea and vomiting that initially occurred three months before admission. Her symptoms started with indigestion, then progressed to nausea, vomiting, bloating and abdominal pain. She presented to an outside hospital, where an obstruction series noted several air fluid levels and haziness suspicious for ascites. A CT abdomen pelvis revealed large ascites, $11 \times 8.7 \mathrm{~cm}$ heterogeneous mass displacing the uterus anteriorly and a $7 \times 5.5 \mathrm{~cm}$ heterogeneous mass in the right adnexa and omental nodularity (Figure 1, 2, and 3). The patient was then referred to a gynecologic oncologist due to suspicion for a gynecologic malignancy and sent to our ER for ongoing

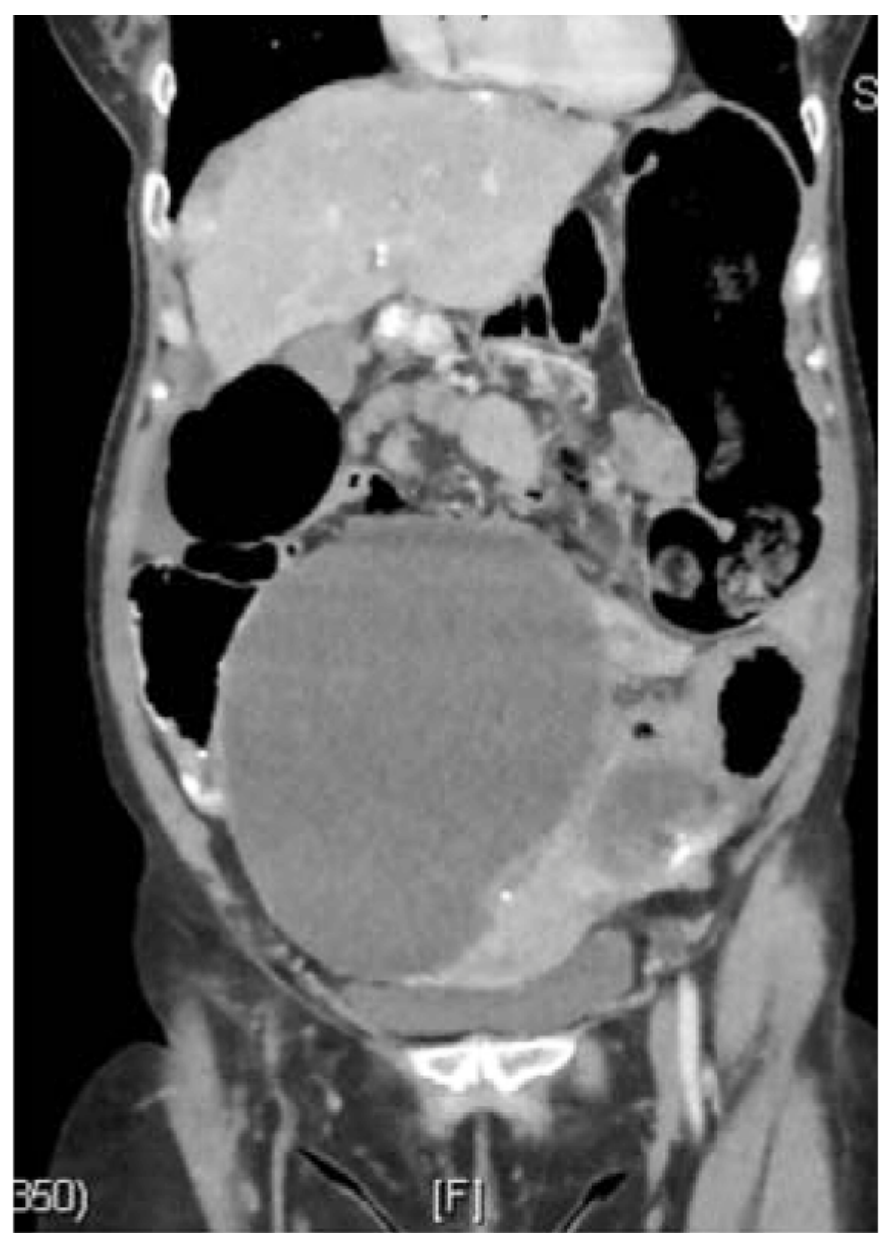

Figure 1: CT scan abdomen and pelvis. Splenic implant present.

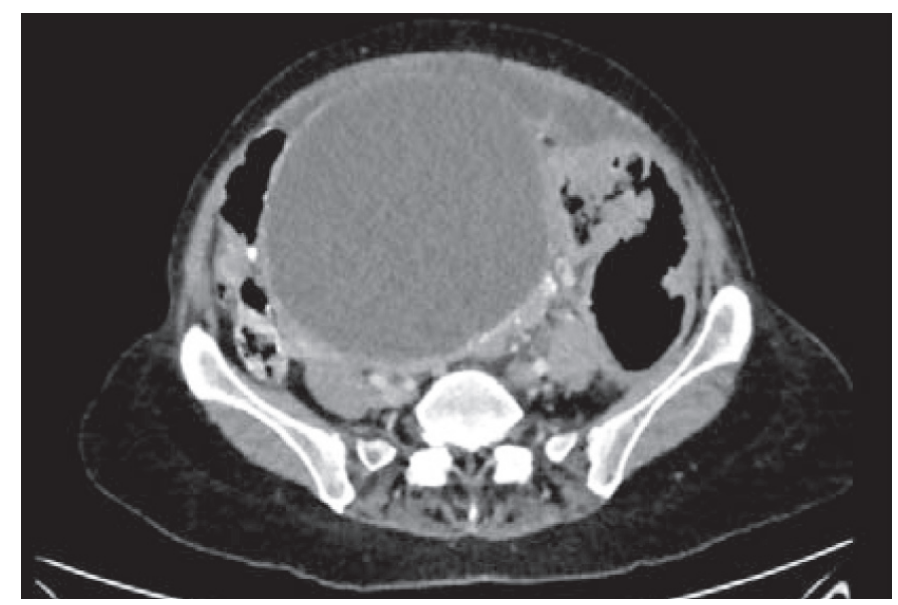

Figure 2: CT scan abdomen and pelvis. Large ovarian mass.



Figure 3: CT scan abdomen and pelvis. $11 \times 8.7 \mathrm{~cm}$ heterogeneous mass displacing the uterus anteriorly.

abdominal pain, nausea, vomiting and bloating.

On pelvic examination, a large mass was noted in the posterior cul-de-sac not fully fixed on sidewall. On digital rectal examination, a large solid mass moderately fixed on sidewall was noted. Laboratory values significant for an elevated CA 125 of 6784.1 (normal o-35) and an elevated CEA of 11.3 (normal 0-2.5). CT chest, abdomen and pelvis revealed no chest metastasis, bilateral adnexal masses with significant sigmoid involvement, omental nodularity, large ascites and a splenic lesion. The patient underwent a diagnostic and therapeutic paracentesis with an FNA and core needle biopsy of the omental mass which revealed well differentiated adenocarcinoma consistent with Mullerian origin. The tumor cells were positive for ER/PR and PAX8.

After appropriate preoperative evaluation and clearance, she was scheduled for primary debulking surgery along with exploratory laparotomy, total abdominal hysterectomy with bilateral salpingo- 


\section{EDORIUM Journals}

oophorectomy, omentectomy, lower anterior resection with colorectal anastomosis, mobilization of splenic flexure, extensive peritoneal resection and bilateral pelvic and paraaortic lymphadenectomy. Both ovaries were enlarged. Right side approximately $6 \mathrm{~cm}$ and complex in appearance.Left side $9 \mathrm{~cm}$ and complex in appearance. There was noted to be diffuse disease with nodularity involving the sigmoid colon and rectum in the posterior cul-de-sac, bilateral surfaces of the diaphragm and several surfaces of bowel mesentery. The masses were sent for frozen section analysis, which was reported to be low-grade serous ovarian carcinoma.

On final pathological examination, the right ovary was $9.8 \mathrm{~cm}$ and left ovary was $13 \mathrm{~cm}$, were both positive for low-grade serous carcinoma arising in a background of borderline tumor with seromucinous features. The uterus, omentum, sigmoid colon, pelvic and paraaortic lymph nodes and umbilicus specimens were all positive for low-grade serous ovarian carcinoma. Pathological staging $\mathrm{T}_{3} \mathrm{~N}_{1} \mathrm{M}_{1}$, consistent with FIGO stage IVB. Immunohistochemical studies revealed ER, PR and WT 1 positivity.

After six weeks post op, patient was started on adjuvant chemotherapy with carboplatin and taxol and has since completed six cycles. She is currently on hormonal therapy with an aromatase inhibitor; letrozole, and has no evidence of disease almost 6 months following completion of chemotherapy.

\section{Case 2}

A 55-year-old female with recurrent Stage IIIA ovarian cancer, initially low malignant potential tumor with micropapillary features which then progressed to lowgrade serous carcinoma. Patient was originally diagnosed with ovarian cancer in January 2009, after experiencing abdominal pain, nausea and vomiting. Since then, patient has had a complicated medical course with multiple hospitalizations throughout the years. Initial imaging revealed bilateral adnexal masses, omental lesion and ascites (Figure 4 and 5). A therapeutic and diagnostic paracentesis was performed and ascites fluid was positive for papillary serous ovarian tumor. A primary debulking surgery including a total abdominal hysterectomy with bilateral salpingo-oophorectomy, omentectomy, bilateral paraaortic and pelvic lymphadenectomy was performed. Initial pathology report revealed a $10 \mathrm{~cm}$ tumor of the right ovary and a $15 \mathrm{~cm}$ tumor of the left ovary, which were both positive for serous borderline tumor with micropapillary features. Small bowel implants and omentum were also positive for serous borderline tumor. Lymph nodes and appendix were negative. She was diagnosed with ovarian cancer with serous borderline tumor stage IIIA and completed six cycles of adjuvant chemotherapy with carboplatin and taxol.

In 2013, she was found to have progression of disease and underwent an exploratory laparotomy resection of multiple mesenteric tumor implants, lower anterior

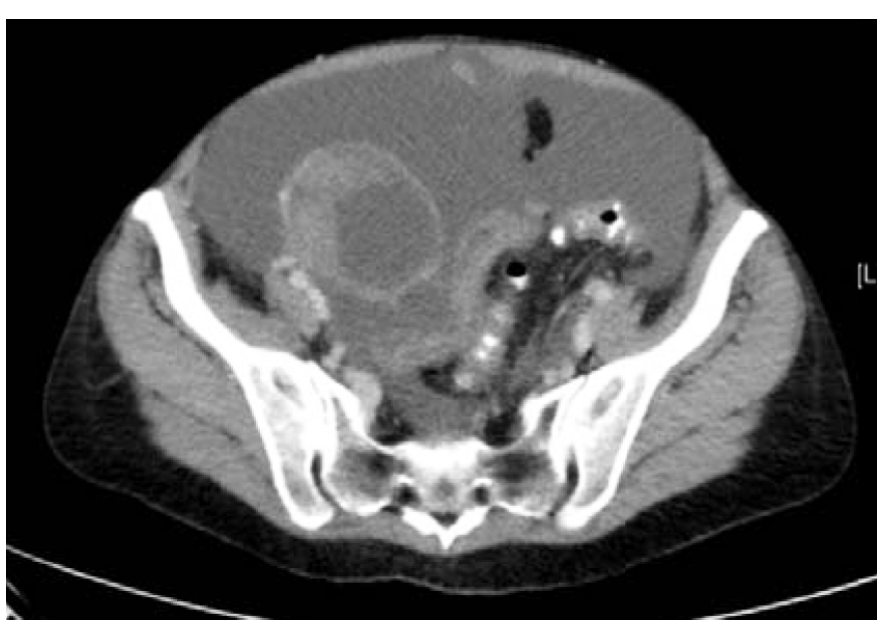

Figure 4: CT scan abdomen and pelvis. Extensive splenic and liver implants.

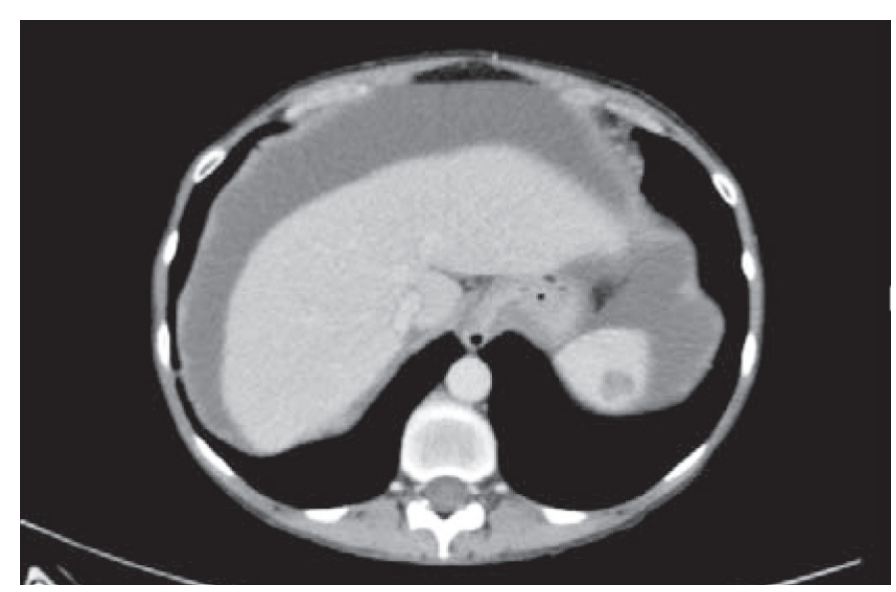

Figure 5: CT scan abdomen and pelvis. Peritoneal implant medial to spleen measuring 24x26 mm.

resection with reanastamosis, peritoneal resection and ablation of diaphragmatic implants. Pathology was significant for low-grade invasive serous carcinoma in multiple sites. Six weeks post op, she started six cycles of intravenous and intraperitoneal cisplatin and taxol.

In 2015, CT scan was again evident for progression of disease. Patient was enrolled in the gynecology oncology group (GOG) 281 clinical trial of the mitogen-activated protein kinase kinase enzymes inhibitor (MEKi), Tramatenib. She tolerated treatment well for about six months, when she was again noted to have progression of disease by CT scan and was ultimately removed from the trial. She then began chemotherapy treatment and received two cycles of intravenous doxorubicin. In June of 2016, she began to experience worsening abdominal pain and was found to have a small bowel obstruction, for which she underwent exploratory laparotomy partial gastrectomy, extended right hemicolectomy, end descending colostomy and suboptimal tumor debulking. In December of 2016, she was found to have erosion of the tumor in the upper rectal stump and underwent treatment with radiation therapy.

In May of 2017, patient was started on weekly 


\section{EDORiUM Journals}

J Case Rep Images Oncology 2018;4:100052Z10JA2018.

www.edoriumjournals.com/case-reports/jcro

Alcid et al. 4

paclitaxel along with bevacizumab up until October, 2017. Patient was hospitalized after noticing feculent drainage from her vagina. Imaging revealed an enterovaginal fistula, however due to the patient's clinical deterioration, was not a surgical candidate for a diverting jejunostomy. She subsequently hospitalized again shortly after discharge, for a deep venous thrombosis. During her last hospitalization, a palliative care and hospice consult was placed, and she ultimately decided to go the home hospice route.

\section{Case 3}

A 58-year-old female with no significant $\mathrm{PMH}$, presented to the ER with acute onset abdominal pain, nausea, vomiting and diarrhea for one day. Patient was in her usual state of health eating and drinking normally, when she started having diffuse abdominal pain. Initially thought it was "gas pain", but progressed to 9/10 pain throughout the day. Her pain was also accompanied by nausea, multiple episodes of vomiting and diarrhea. A CT abdomen pelvis revealed large bilateral pelvic masses obstructing the colon and ureters (Figure 6 and 7). Soft tissue nodules and ascites also present. Findings were most concerning for peritoneal carcinomatosis (Figure 8 and 9).

Pelvic examination revealed a firm, fixed mass in pelvis with nodularity noted in cul-de sac. Laboratory values significant for a CA 125 of 5121.2. Due to her clinical deterioration and findings of bowel obstruction on CT scan, she underwent exploratory laparotomy with diverting colostomy. At this time, it was decided that patient was not stable enough for cytoreductivedebulking, and will undergo omental biopsy and aspiration of ascites fluid to establish diagnosis. After completion of her transverse loop colostomy, she then underwent resection of the omental nodule and aspiration of ascites. The omental nodule was $5.5 \times 3 \mathrm{~cm}$ in size and was sent to pathology for frozen section analysis. Results were positive for low-grade serous carcinoma and ascites fluid positive for adenocarcinoma.

Patient was started on neoadjuvant chemotherapy with carboplatin and taxol, completed three cycles, and then ultimately underwent TAH, BSO, radical tumor debulking, total protocolectomy, appendectomy, splenectomy and terminal ileostomy. Final pathologic examination of the ovaries was significant for low-grade serous carcinoma, which was also noted to involve the uterus, vagina, serosa of the colon with invasion to submucosa, falciform ligament, diaphragm and spleen. Pathological staging pT3c No M1, consistent with FIGO stage IVB. After successful surgery, she was started on hormonal therapy with letrozole. Unfortunately, follow up imaging revealed increasing nodular peritoneal thickening suspicious for progressive peritoneal carcinomatosis. She was continued on hormonal therapy and was open to enrolling in a clinical trial.



Figure 6: CT scan abdomen and pelvis. Large bilateral pelvic masses.

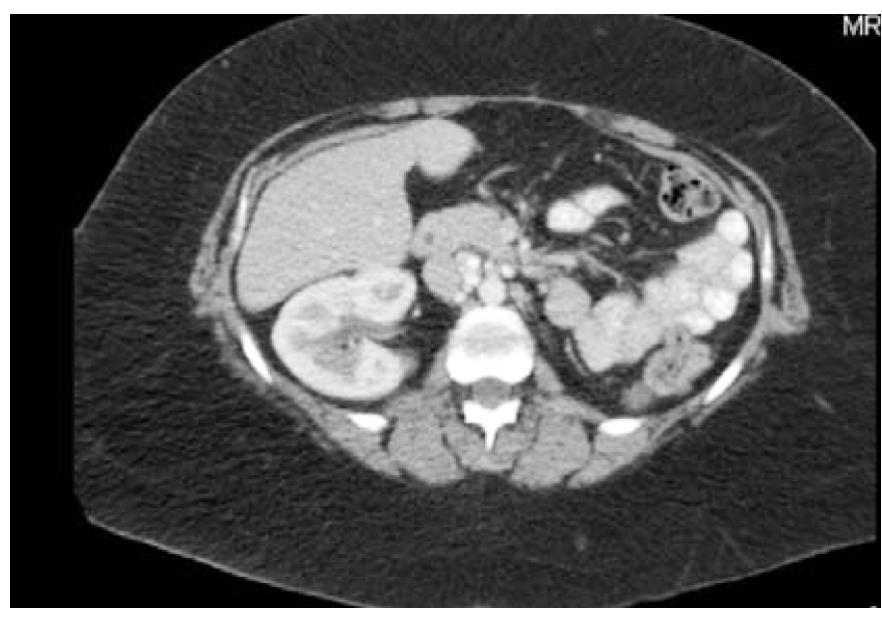

Figure 7: CT scan abdomen and pelvis coronal view. Large bilateral pelvic masses obstructing the colon and ureters.



Figure 8: CT scan abdomen and pelvis. Multiple liver and splenic implants.

\section{Case 4}

A 31-year-old female with recurrent stage IIIC borderline serous ovarian carcinoma initially diagnosed 


\section{EDORIUM Journals}

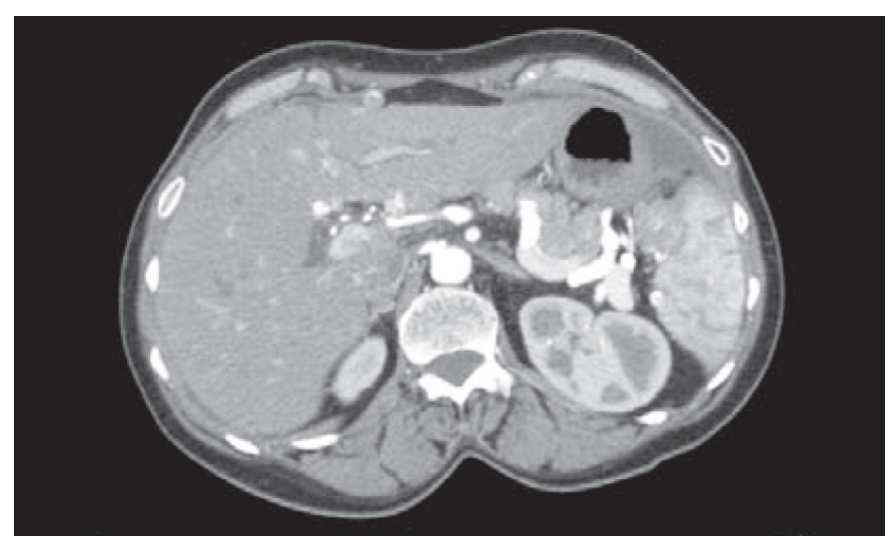

Figure 9: CT scan abdomen and pelvis. Peritoneal implants suggestive of carcinomatosis.

at 24 years of age. Patient has a $\mathrm{PMH}$ significant for bicornate uterus and solitary kidney, otherwise a healthy young adult. She initially presented to the ER complaining of abdominal pain, nausea, bloating and indigestion that started three months prior. CT abdomen pelvis revealed a $10.8 \times 8.8 \times 7.9 \mathrm{~cm}$ right ovarian mass, $10 \times 13 \times 11.8 \mathrm{~cm}$ left ovarian mass, large amount of ascites and omental inflammation. A paracentesis was performed which revealed cohesive papillary-like groups of abnormal cells consistent with neoplasm of gynecologic origin.

She underwent bilateral salpingo-oophorectomy, omentectomy, pelvic and para-aortic lymphadenectomy, resection of tumor implants of the bowel and colon, with optimal cytoreduction. Official pathologic tissue diagnosis consistent with borderline serous ovarian carcinoma, pT3 N1 MO, consistent with FIGO stage IIIC. Patient ultimately had a total abdominal hysterectomy and resection of multiple retroperitoneal lymph nodes including low and high periaortic, inter aortocaval, celiac trunk, hepatic artery and retropancreatic lymph nodes. There was evidence of metastatic low-grade serous carcinoma involving multiple matted lymph nodes (Figure 10 and 11).

She then completed six cycles of adjuvant chemotherapy with taxol and carboplatin. She ultimately had progression of disease as evident by the innumerable pulmonary nodules and hepatic lesions. She is currently receiving hormonal therapy treatment with fulvestrant monthly and awaiting possible entry into a clinical trial with a MEK inhibitor.

\section{DISCUSSION}

Serous carcinoma represents the most common histological subtype of epithelial ovarian cancer. The serous subtype can be further characterized by high-grade (90\%) and low-grade (10\%). The hallmarks of this disease include young age at diagnosis, relative chemoresistance compared to high-grade serous carcinoma of the ovary.

Histologic grade has been found to be a major

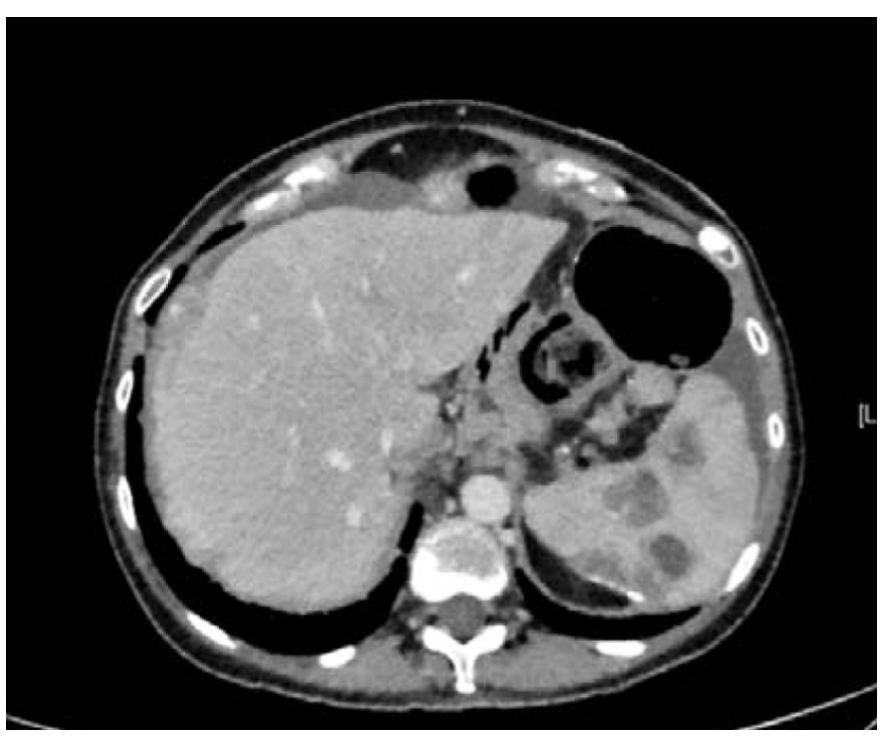

Figure 10: CT scan abdomen and pelvis. Enlarged matted portocaval lymph node $1.7 \mathrm{cmx} 2.6 \mathrm{~cm}$.

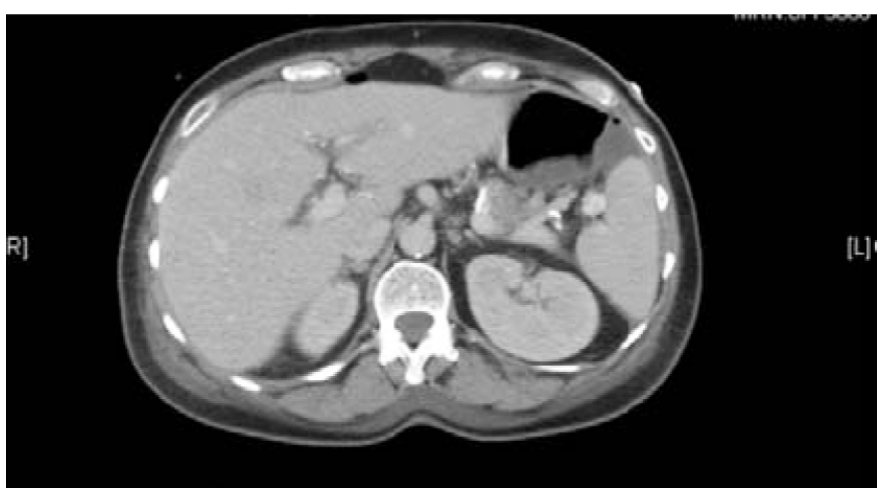

Figure 11: CT scan abdomen and pelvis. Enlarged lymph node $1 . \mathrm{cm} \times 1.5 \mathrm{~cm}$ between the aorta and inferior vena cava.

prognostic factor in these patients, however, no universal grading system exists. Investigators at MD Anderson Cancer Center proposed a two-tier grading system for serous carcinoma of the ovary, and a recent gynecologic oncology group (GOG) study determined this binary system to be more superior to that of the International Federation of Gynecology and Obstetrics (FIGO) grading system [1].

Low-grade serous carcinoma may arise following an initial diagnosis of serous tumor of low malignant potential (serous borderline tumor), which strongly supports a developmental relationship [1]. The potential model of disease progression includes benign cysts to borderline tumors then to invasive low-grade serous carcinoma. There are multiple molecular features that distinguish the low-grade serous carcinoma from the high-grade carcinomas. The low-grade serous carcinomas have a much lower frequency of p53 mutations, greater expression of estrogen receptor (ER) and progesterone receptor (PR), and also mutations in KRAS and BRAF [2-6]. 


\section{EDORiUM Journals}

Clinical presentation of low-grade serous carcinoma of the ovary are similar to those of the other epithelial cancers of the ovary, which may include, an asymptomatic adnexal mass, to bloating, early satiety, urinary urgency and abdominal or pelvic pain. Approximately 90\% of patients will present with advanced disease, stage II to IV. The median age at diagnosis is 43 to 46.5 years of age $[7,8]$.

The primary modality of treatment for these patients is surgery. For patients with stage II-IV low-grade serous carcinoma, the standard is maximum cytoreductive surgery, with the goal of achieving minimal residual disease. In an ancillary study of GOG 182, Fader et al, 189 women with grade 1 (low-grade) serous carcinoma were studied and the conclusion was that residual disease status following primary surgery was associated with overall survival (OS). Patients with microscopic residual disease had significantly longer median progression-free survival (PFS)(33.2 months) and OS (96.6 months) compared with those of gross $0.1 \mathrm{~cm}-1 \mathrm{~cm}$ residual disease (14.1 and 44.5 months, respectively) [9]. Following surgery, adjuvant chemotherapy with platinum/taxane regimen is often administered, typically for six cycles. Unlike the high-grade serous type, intraperitoneal chemotherapy has not been shown to be more beneficial, over the standard chemotherapy. In a recent report from the Arbeitsgemeinschaft Gyneaekolgische Onkologie (AGO) study group database of 5,114 patients, found that the response rate to platinum/taxane chemotherapy was $23 \%$ after suboptimal debulking, compared to $90 \%$ response in the high-grade serous carcinoma patients who have been sub optimally debulked $[10,11]$. The overall conclusion was that low-grade serous carcinoma is not as responsive to standard chemotherapy as highgrade serous carcinoma.

Although low-grade serous carcinoma of the ovary carries a relatively good prognosis, over $80 \%$ of patients will experience disease recurrence. Secondary cytoreductive surgery may be considered in selected patients, particularly those who have platinum sensitive disease and with limited metastatic sites. Systemic therapy options for salvage therapy include chemotherapy, hormonal therapy and targeted agents.

Hormonal therapy is also an option for patients with recurrent disease, as a high proportion of these tumors are estrogen receptor (ER) positive. In a recent study from The University of Texas MD Anderson Cancer Center reported by Dr. David Gershenson, found that hormonal therapy reduced the risk of recurrence by $77 \%$ compared with surveillance [10]. Although there is a much greater frequency of persistent disease after primary treatment, patients receiving hormonal therapy as maintenance had less disease progression. Median progression-free survival was 27.3 months with surveillance and 64.9 months with hormonal therapy. In patients who did not have any evidence of disease after primary treatment, median progression-free survival was 29.9 months with surveillance and 81.1 months with maintenance hormonal therapy $[10,11]$.

Since systemic chemotherapy has limited activity in low-grade serous carcinoma, other forms of more effective systemic treatment such as targeted therapies are currently being studied. The mitogen-activated protein kinase pathway (MAPK), is known to be involved in the pathogenesis of low malignant potential and lowgrade serous carcinomas, based on Singer et al [12] The MEK enzyme has been the target for therapy, and a number of MEK inhibitors (MEKi) have been developed over the past few years [13,14]. In a GOG 0239 trial with MEKiselumentinib, reported by Farley et al. [15], provided promising results. In a study of fifty-two women with recurrent low grade-serous carcinoma, the overall response rate was $15 \%$ with one complete response and seven partial responses. Angiogenesis inhibitors such as bevacizumab have also been studied. According to the ICON 7 trial, patients with low-grade serous carcinoma were treated with standard chemotherapy with or without bevacizumab, and results show there was no benefit with the addition of bevacizumab to standard chemotherapy for first line treatment [16]. However, for patients with recurrent low-grade serous carcinoma, based on a single institution study by Dalton HJ et al., 45 women who received bevacizumab regimens had a response rate of $48 \%$ [17].

\section{CONCLUSION}

Low-grade serous carcinoma of the ovary may occur de novo or following a diagnosis of serous tumor of low malignant potential. Most patients will present with advanced disease (stage II-IV). The primary treatment is cytoreductive surgery, followed by platinum/taxane chemotherapy. Due to the expression of estrogen receptors on these tumors, hormonal treatment has been shown to decrease the risk of recurrence compared with surveillance. Targeted therapy for recurrent disease has also shown to have promising results.

\section{REFERENCES}

1. Malpica A, Deavers MT, Lu K, et al. Grading ovarian serous carcinoma using a two-tier system. Am J Surg Pathol 2004 Apr;28(4):496-504.

2. Teneriello MG, Ebina M, Linnoila RI, et al. p53 and Kiras gene mutations in epithelial ovarian neoplasms. Cancer Res 1993 Jul 1;53(13):3103-8.

3. Anglesio MS, Arnold JM, George J, et al. Mutation of ERBB2 provides a novel alternative mechanism for the ubiquitous activation of RAS-MAPK in ovarian serous low malignant potential tumors. Mol Cancer Res 2008 Nov;6(11):1678-90.

4. Wong KK, Tsang YT, Deavers MT, et al. BRAF mutation is rare in advanced-stage low-grade ovarian serous carcinomas. Am J Pathol 2010 Oct;177(4):1611-7.

5. Meinhold-Heerlein I, Bauerschlag D, Hilpert F, et al. Molecular and prognostic distinction between serous 


\section{EDORIUM Journals}

ovarian carcinomas of varying grade and malignant potential. Oncogene 2005 Feb 3;24(6):1053-65.

6. Bonome T, Lee JY, Park DC, et al. Expression profiling of serous low malignant potential, low-grade, and high-grade tumors of the ovary. Cancer Res 2005 Nov 15;65(22):10602-12.

7. Gershenson DM, Sun CC, Lu KH, et al. Clinical behavior of stage II-IV low-grade serous carcinoma of the ovary. Obstet Gynecol 2006 Aug;108(2):361-8.

8. Gershenson DM, Bodurka DC, Lu KH, et al. Impact of age and primary disease site on outcome in women with low-grade serous carcinoma of the ovary or peritoneum: Results of a large single-institution registry of a rare tumor. J Clin Oncol 2015 Aug 20;33(24):2675-82.

9. Fader AN, Java J, Ueda S, et al. Survival in women with grade 1 serous ovarian carcinoma. Obstet Gynecol 2013 Aug;122(2 Pt 1):225-32.

10. Gershenson DM, Bodurka DC, Coleman RL, Lu KH, Malpica A, Sun CC. Hormonal maintenance therapy for women with low-grade serous cancer of the ovary or peritoneum. J Clin Oncol 2017 Apr 1;35(10):110311.

11. Grabowski JP, Harter P, Heitz F, et al. Operability and chemotherapy responsiveness in advanced lowgrade serous ovarian cancer. An analysis of the AGO study group metadatabase. Gynecol Oncol 2016 Mar;140(3):457-62.

12. Singer G, Oldt R 3rd, Cohen Y, et al. Mutations in BRAF and KRAS characterize the development of low-grade ovarian serous carcinoma. J Natl Cancer Inst 2003 Mar 19;95(6):484-6.

13. Neuzillet C, Tijeras-Raballand A, de Mestier L, Cros J, Faivre S, Raymond E. MEK in cancer and cancer therapy. Pharmacol Ther 2014 Feb;141(2):160-71.

14. Miller CR, Oliver KE, Farley JH. MEK1/2 inhibitors in the treatment of gynecologic malignancies. Gynecol Oncol 2014 Apr;133(1):128-37.

15. Farley J, Brady WE, Vathipadiekal V, et al. Selumetinib in women with recurrent low-grade serous carcinoma of the ovary or peritoneum: An open-label, single-arm, phase 2 study. Lancet Oncol 2013 Feb;14(2):134-40.

16. Oza AM, Cook AD, Pfisterer J, et al. Standard chemotherapy with or without bevacizumab for women with newly diagnosed ovarian cancer ( $\left.\mathrm{ICON}_{7}\right)$ :
Overall survival results of a phase 3 randomised trial. Lancet Oncol 2015 Aug;16(8):928-36.

17. Dalton HJ, Fleming ND, Sun CC, Bhosale P, Schmeler KM, Gershenson DM. Activity of bevacizumabcontaining regimens in recurrent low-grade serous ovarian or peritoneal cancer: A single institution experience. Gynecol Oncol 2017 Apr;145(1):37-40.

$* * * * * * * * *$

\section{Author Contributions}

Joel Alcid - Substantial contributions to conception and design, Acquisition of data, Drafting the article, Revising it critically for important intellectual content, Final approval of version to be published

Mark S. Shahin - Substantial contributions to conception and design, Acquisition of data, Drafting the article, Revising it critically for important intellectual content, Final approval of version to be published

\section{Guarantor of Submission}

The corresponding author is the guarantor of submission.

\section{Source of Support}

None

\section{Consent Statement}

Written informed consent was obtained from the patient for publication of this case series.

\section{Conflict of Interest}

Authors declare no conflict of interest.

\section{Copyright}

(C) 2018 Joel Alcid et al. This article is distributed under the terms of Creative Commons Attribution License which permits unrestricted use, distribution and reproduction in any medium provided the original author(s) and original publisher are properly credited. Please see the copyright policy on the journal website for more information.
Access full text article on other devices



Access PDF of article on other devices

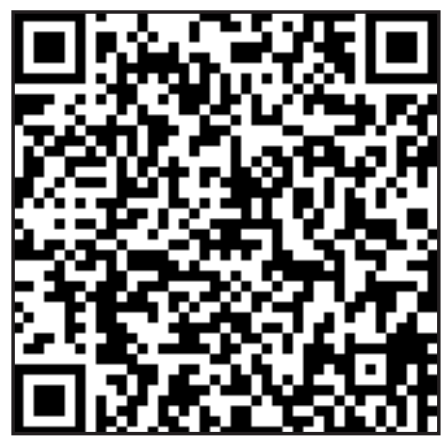

